\title{
ARTICLE
}

\section{EGFR activation-induced decreases in claudin1 promote MUC5AC expression and exacerbate asthma in mice}

\author{
Zhirong Jia $\mathbb{D}^{1,2}$, Kaifan Bao ${ }^{1}$, Pan Wei ${ }^{1}$, Xuerui $\mathrm{Yu}^{1}$, Yuheng Zhang ${ }^{1}$, Xiaotong Wang ${ }^{1}$, Xiaoyu Wang ${ }^{1}$, Lu Yao ${ }^{1}$, Lianqu $\mathrm{Li}^{1}, \mathrm{Peng} \mathrm{Wu}^{1}$,
} Weiyuan Yuan ${ }^{1}$, Siqi Wang ${ }^{1}$, Jie Zheng ${ }^{1,3}$, Yongqing Hua ${ }^{1}$ and Min Hong ${ }^{1}$

Claudin 1 plays a critical role in maintaining the epithelial barrier, and mucus hypersecretion induced by epidermal growth factor receptor (EGFR) activation is a pivotal pathological feature of asthma. The relationship between claudin1 expression and mucus hypersecretion and EGFR activation is still poorly understood. In this report, we showed that claudin1 expression correlated with asthma stage, in both patients with asthma and in the house dust mite (HDM)-induced mouse asthma model. Claudin1 knockdown induced MUC5AC overexpression both in 16HBE cells and in mouse airways. In addition, claudin 1 expression negatively correlated with asthma severity as demonstrated by significantly higher MUC5AC expression, more severe airway inflammation, and increased airway hyperreactivity in mouse lungs with claudin1 knockdown following HDM challenge. EGFR activation reduced claudin 1 expression and increased MUC5AC expression, both in vitro and in vivo. Erlotinib alleviated murine allergic airway inflammation, restored claudin 1 expression and decreased MUC5AC expression. These results suggest that EGFR activation-induced decreases in claudin 1 promote goblet-cell metaplasia, and restoring claudin 1 to maintain barrier integrity by EGFR antagonism may provide a novel therapeutic strategy for asthma.

Mucosal Immunology (2021) 14:125-134; https://doi.org/10.1038/s41385-020-0272-z

\section{INTRODUCTION}

Asthma, a common chronic airway inflammatory disease, affects more than 300 million people worldwide. The clinical manifestations of asthma are caused by obstruction of the conducting airways of the lung, and bronchial hyperresponsiveness ${ }^{1}$. One of the characteristics of asthma, mucus abnormalities including mucin overproduction and hypersecretion, contribute to airway obstruction ${ }^{2-4}$. Notably, airway mucus plugging has long been recognized as a principal cause of death in asthma patients ${ }^{5}$. However, the molecular mechanisms of mucin hypersecretion have not been comprehensively investigated until recently.

Altered airway epithelial barrier function might be one potential mechanism of mucus production. As the front line of defense against inhaled pathogens and particles, airway epithelial cells could initiate airway inflammation and produce mucus, which are increasingly recognized as important contributors to airway obstruction in allergic asthma ${ }^{6}$. More importantly, airway epithelial cells form a tight barrier against environmental stimuli via tight junctions (TJs) and adherens junctions (AJs). Increasing evidence indicates that defects in $\mathrm{TJ}$ and $\mathrm{AJ}$ proteins are a feature of airway inflammation in asthma, which may cause changes in epithelial morphology and integrity, potentially leading to faster trafficking of inflammatory cells through the epithelium ${ }^{7,8}$. Moreover, in asthma, several reports have shown that epithelial susceptibility to injury and repair responses are abnormal ${ }^{9,10}$. Considering that asthma is highly associated with disrupted epithelial cell-cell junctions, impaired barrier function, and excessive mucus production, we speculate that loss of an epithelial barrier protein might directly regulate goblet-cell metaplasia.

Recent reports suggest that TJs participate in signal transduction mechanisms that regulate epithelial cell proliferation, gene expression, differentiation, and morphogenesis ${ }^{11}$. Claudins, one family of TJ proteins, are expressed in the epithelia and form paracellular barriers and pores that determine TJ permeability ${ }^{12}$, and alteration of claudin expression plays an important role in asthma ${ }^{13}$. Moreover, it has been reported that claudins are directly related to epithelial differentiation ${ }^{14}$. Given that goblet-cell metaplasia represents abnormal epithelial differentiation, which promotes excessive mucus secretion ${ }^{15}$, and claudin 1 is indispensable in TJ structure and maintaining epithelial barrier ${ }^{16}$, a relationship may exist between claudin1 and mucus over secretion. Thus, the regulation of claudin 1 expression and function is worthy of study.

One well-recognized feature of epithelial alterations in asthmatic airway is increased expression of epidermal growth factor receptor (EGFR) and several of its ligands, leading to corticosteroid-insensitive EGFR activation ${ }^{17,18}$. Growing evidence has demonstrated that multiple stimuli lead to hypersecretion of mucins (especially MUC5AC/Muc5ac, a marker for goblet cells) via EGFR expression and activation, causing gobletcell metaplasia by cell differentiation ${ }^{19-21}$. Expressions of EGFR and MUC5AC are both prominently upregulated in the epithelia of asthmatic patients, and colocalize in goblet cells ${ }^{22,23}$.

\footnotetext{
1Jiangsu Key Laboratory for Pharmacology and Safety Evaluation of Chinese Materia Medica, School of Pharmacy, Nanjing University of Chinese Medicine, 210046 Nanjing, China; ${ }^{2}$ School of Basic Medicine and Clinical Pharmacy, China Pharmaceutical University, 211198 Nanjing, China and ${ }^{3}$ School of Medicine and Life Science, Nanjing University of Chinese Medicine, 210046 Nanjing, China

Correspondence: Min Hong (minhong@njucm.edu.cn)

These authors contributed equally: Zhirong Jia, Kaifan Bao
}

Received: 3 June 2019 Revised: 9 February 2020 Accepted: 11 February 2020

Published online: 4 March 2020 
126

Moreover, inhibition of EGFR signaling reduces goblet-cell metaplasia and airway hyperreactivity (AHR) in the house dust mite (HDM)-induced asthma model ${ }^{24}$. These studies suggest that EGFR expression and activation, especially in the airway epithelium, are of great significance in mediating goblet-cell metaplasia and AHR in asthma. We speculate that EGFR activation may regulate goblet-cell metaplasia and AHR via regulating claudin 1 .

\section{RESULTS}

Claudin 1 expression correlates with the stage of asthma According to the primary data from the NCBI GEO database (GSE43696), the normalized expressions of genes encoding AJ protein (CDH1), TJ proteins (TJP1, TJP2, OCLN, CLDN1-12, CLDN1419, CLDN22, CLDN23, CLDND1, and CLDND2), Desmoglein (DSG) proteins (DSG 1-4), and gap junction (GJ) proteins (GJA1, GJA3, GJA4, GJB1, GJB2, GJB3, and GJC3) in bronchial epithelial cells from normal (control, $n=20$ ) and patients with mild asthma $(n=50)$ or severe asthma $(n=38)$ were analyzed (Fig. S1a). We identified four differentially expressed mRNAs (CLDN1, CLDN10, GJB1, and GJB3) in control and mild asthma (Fig. S1b) and six differentially expressed mRNAs (CLDN1, CLDN16, CLDN23, $C L D N 10, G J B 1$, and GJC3) in control and severe asthma (Fig. S1c), and the results showed that CLDN1 expression was reduced significantly both in bronchial epithelial cells from patients with mild and severe asthma, when compared with normal patients $(P=1.63 \mathrm{E}-02$, and $P=6.27 \mathrm{E}-03$, respectively; Fig. S1d). To examine claudin 1 expression at different stages of asthma in the HDM-induced animal model, mice were first sensitized to HDM by three intraperitoneal injections followed by three HDM administrations intranasally. One group of mice was euthanized after the first round of allergen exposures (EI), while another group were rested and allowed to recover for 16 days. Mice were re-exposed to HDM intranasally three times and euthanized $24 \mathrm{~h}$ after the last HDM exposure (EII) (Fig. 1a). Our results showed that airway resistance in the Ell group was significantly higher than that in El group (data not shown). The severity of inflammatory infiltration around the trachea in the Ell group was much worse than that in the El group (Fig. 1b). Moreover, the levels of IL-4 and IL-13 in BALF of the Ell group were also higher than that of the El group (Fig. 1c). More importantly, we observed significant downregulation of claudin 1 both in the El and Ell groups, plus a remarkable decrease of claudin 1 in Ell compared with that in El (Fig. 1d, e). These results suggested that claudin1 was significantly decreased after HDM exposure and correlated with the stage of asthma.

Knockdown of claudin 1 promotes MUC5AC expression

The 16HBE cells were cultured with endogenous claudin1 inhibition by selecting a small hairpin RNA (shRNA) targeting the common region of CLDN1 (Fig. 2a). The results indicated that several shRNA sequences (shCLDN1-3, -4, -5, -6) could effectively reduce the claudin 1 transcript and protein (Fig. 2b). Western blots and gene expression analyses suggested that claudin 1 knockdown (shCLDN1) promoted upregulation of MUC5AC (Fig. 2c, d). Immunofluorescence analysis further demonstrated that claudin 1 knockdown resulted in a remarkable increase in the number of cells expressing MUC5AC (Fig. 2e), suggesting a role for claudin1 in promoting goblet-cell metaplasia. Moreover, CLDN1 downregulation (Fig. S2a) in 16HBE cells increased the levels of TSLP (Fig. S2b, c); however, it had no significant effect on IL-33 expression (Fig. S2d). Furthermore, 16HBE cells were transfected with control shRNA or shCLDN1 and then differentiated for 28 days utilizing ALI cultures. The results showed that CLDN1 mRNA was significantly decreased by shCLDN1 (Fig. 2f), which remarkably increased MUC5AC expression (Fig. $2 \mathrm{~g}$ ) and reduced the TEER, indicating reduced barrier function (Fig. $2 \mathrm{~h}$ ). In contrast, the mRNA levels of $C D H 1$ and OCLN had no detectable change (Fig. S3a, b). These results suggested that claudin 1 was pivotal for epithelial barrier function and downregulation of this TJ might promote goblet-cell metaplasia.

\section{Knockdown of claudin1 expression exacerbates HDM-induced} asthma

Next, to determine the role of claudin1 in asthma, adenoassociated virus (AAV2/5)-shRNA-claudin1 vectors were generated and screened (Fig. S4) to knockdown claudin1 expression in murine lung by intratracheal injection in the HDM-induced asthma model (Fig. 3a). At day 21 after AAV injection, the infection efficiency was detected (Fig. S5), and we observed a significant decrease of claudin1 expression in AAV-shRNA-claudin1-infected lung bronchial epithelial cells (Fig. S6a, b). Furthermore, abnormal morphology and inflammation around the bronchovascular bundle, and increased expression of MUC5AC were observed in mice treated with shCLDN1 (Fig. S6c-e). Mice were then exposed to HDM to evaluate the effect of claudin 1 knockdown. The results suggested that claudin 1 silencing (Fig. $3 \mathrm{~b}$ ) in murine lung led to an increased number of eosinophil (EOS) cells in peripheral blood and inflammatory cells in BALF (Fig. 3c, d), as well as increased inflammatory infiltration around the bronchovascular bundle (Fig. 3e) and elevated concentrations of IL-4, IL-5, and IL-13 in BALF (Fig. 3f). Furthermore, increases of MUC5AC (Fig. 3g, h) and mucus secretion (Fig. S7) were evident in airway epithelial cells of asthmatic mice infected with AAV-shRNA-claudin1, and claudin1 knockdown significantly augmented AHR induced by HDM challenge (Fig. 3i). These results suggested that the downregulation of claudin 1 exacerbated the development of asthma.

EGFR activation promotes MUC5AC expression by downregulating claudin1

Among several stimulators, EGF and HBEGF were the most significant factors in decreasing the expression of claudin1 compared with HDM, LPS, and TNF-a (Fig. 4a). Increasing concentrations of HBEGF (0-40 ng/mL) (Fig. 4b) or EGF (0-40 ng/ $\mathrm{mL}$ ) (Fig. S8a) caused a dose-dependent decrease of claudin1 expression in 16HBE after a $24 \mathrm{~h}$ treatment period, but did not significantly affect protein levels of E-cadherin, ZO-1, or occludin. There was a sharp time-dependent drop of claudin 1 expression in $16 \mathrm{HBE}$ between 12 and $24 \mathrm{~h}$ (Fig. 4c, S8b). This trend was further confirmed by immunofluorescence analyses (Fig. 4d). In subsequent experiments, we found that IL-13 significantly reduced the expression of claudin 1 at 60 and $100 \mathrm{ng} / \mathrm{mL}$ (Fig. 4e), and the combination of HBEGF and IL-13 together downregulated claudin 1 expression (Fig. 4f, and S8c). In addition, tyrphostin AG1478 , a selective EGFR inhibitor, reversed the decreased expression of claudin 1 induced by HBEGF or IL-13 (Fig. 4g, h).

HBEGF treatment resulted in a remarkable increase in the number of cells expressing MUC5AC (Fig. 4i), and simultaneously, in MUC5AC protein levels (Fig. 4j). Consistent with these results, HBEGF induced the mRNA expression of MUC5AC in 16HBE cells (Fig. 4k) and in ALI cultures (Fig. 4l). Moreover, in ALI cultures, HBEGF substantially reduced the TEER (Fig. $4 \mathrm{~m}$ ) and significantly decreased mRNA levels of CLDN1 (Fig. 4n), whereas negligible effects were observed on $C D H 1$ and OCLN mRNAs (Fig. 4o). Furthermore, we overexpressed claudin 1 in 16HBE cells by transfecting with the pc-DNA3.1-CLDN1 plasmid, and found that MUC5AC upregulation induced by HEBGF was reversed by claudin 1 overexpression (Fig. 4p). Overall, these results demonstrated that EGFR activation reduced claudin1 expression, which contributed to epithelial barrier disruption and goblet-cell metaplasia.

Erlotinib restores claudin 1 expression and inhibits MUC5AC expression in HDM-induced mice asthma

Erlotinib, a tyrosine kinase inhibitor that antagonizes EGFR activation, was administered during the HDM treatment period 


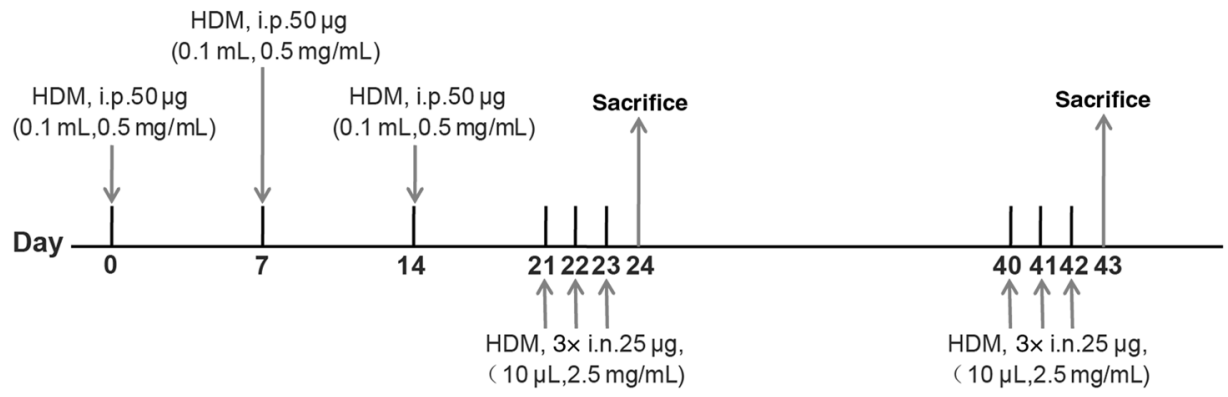

b
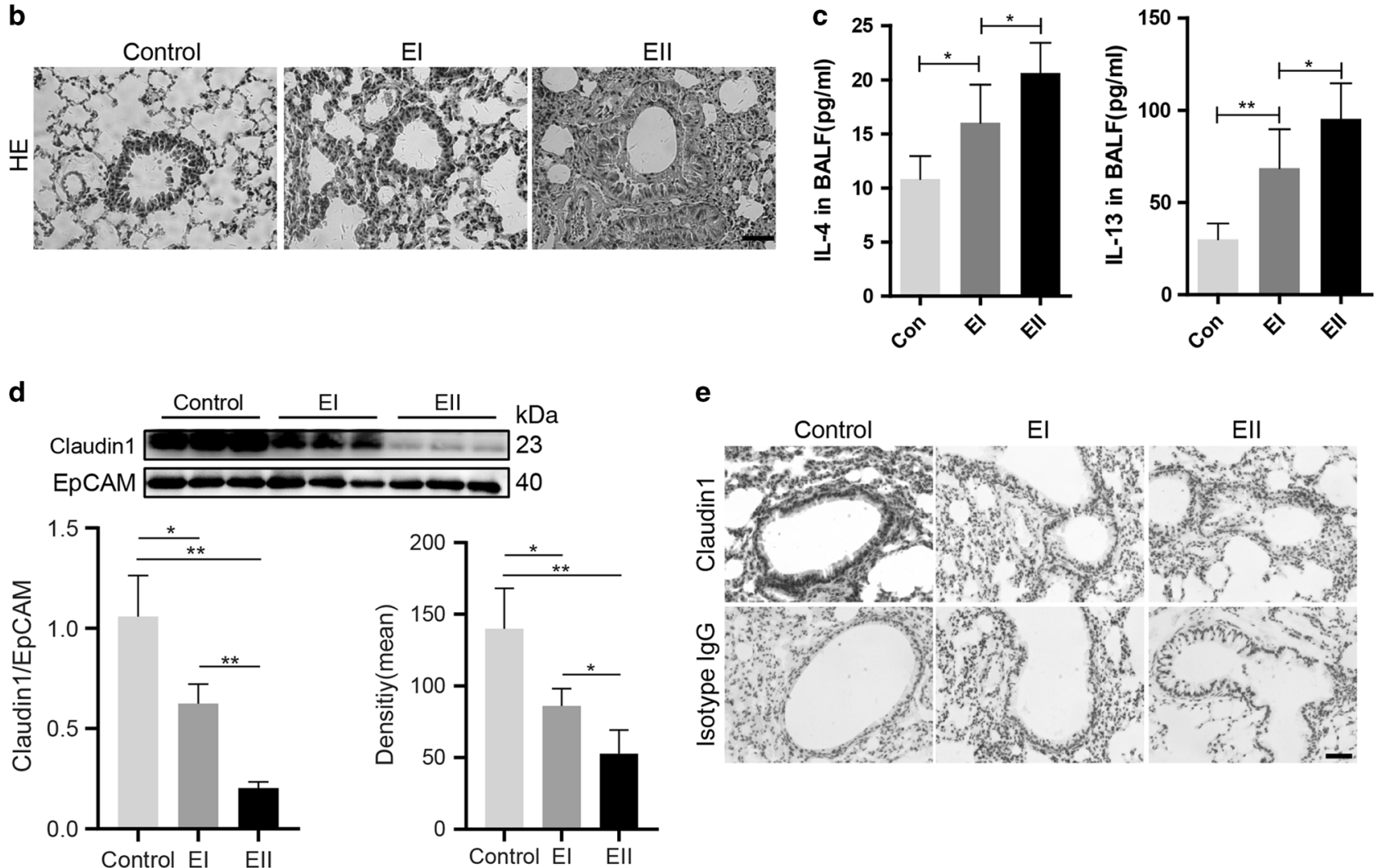

e

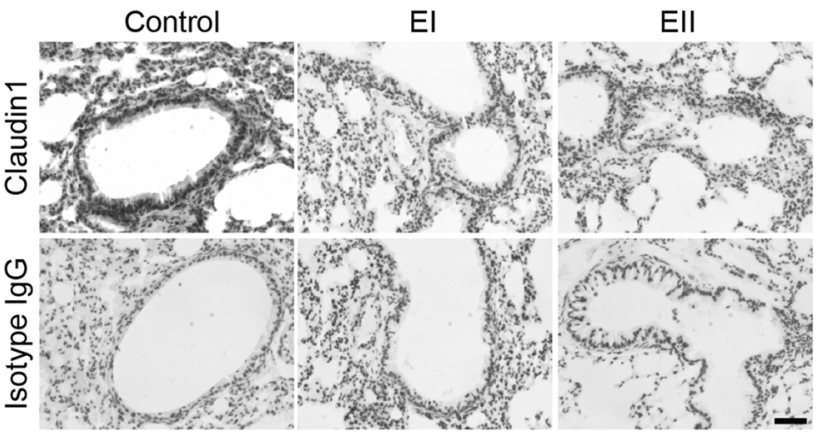

Fig. 1 Claudin 1 is significantly downregulated in the HDM-induced asthma animal model. a Flow charts of the HDM-induced asthma mouse model. b Hematoxylin and eosin -stained lung tissue sections. (Scale bar: $50 \mu \mathrm{m}$; original magnification: $\times 400 ; n=3$ ). c The levels of IL-4 and IL-13 in BALF were detected by ELISA $(n=6)$. $\mathbf{d}$ The expression of claudin1 in each period of asthma was detected by western blotting. The data are expressed as the mean \pm SD of the ratios of claudin 1 to EpCAM, $n=6$. e The expression of claudin 1 and its isotype lgG in each period of asthma was detected by immunohistochemistry and the mean density of claudin 1 was analyzed by IPP software (scale bar: $50 \mu$ m; original magnification: $\times 400 ; n=3$ ). El-M: the model of first excitation. Ell-M: the model of second excitation. Statistical comparisons were performed using one-way analysis of variance with Dunnett's test and unpaired two-tailed Student's $t$ - test with Welch's correction ${ }^{* * *} P<$ $0.001 ;{ }^{*} P<0.01 ; * P<0.05$; mean $\left.\pm \mathrm{SD}\right)$.

(Fig. 5a). Immunostaining results showed that p-EGFR increased in mice lung after HDM exposure, especially in bronchiolar epithelial cells, which could be inversely attenuated by EGFR inhibition (Fig. 5b). AHR was observed in all HDM-treated mice after elicitation by $25 \mathrm{mg} / \mathrm{mL}$ and $50 \mathrm{mg} / \mathrm{mL}$ methacholine, whereas these responses were remarkably attenuated in mice treated with erlotinib (Fig. 5c). The HDM-treated group showed increases in total cell counts in BALF and EOS counts in peripheral blood, and EGFR inhibition could block inflammatory cell influx (Fig. $5 \mathrm{~d}$, e). Histological analyses showed that bronchial wall thickening and inflammatory infiltration around the trachea induced by HDM were attenuated by erlotinib treatment (Fig. 5f). Th2 cytokines IL-4 and IL-13 in BALF were also reduced to a certain extent by erlotinib treatment (Fig. 5g). Furthermore, in the HDM-induced asthma model, erlotinib restored the expression of claudin 1 and decreased the expression of MUC5AC (Fig. 5h) and mucus secretion (Fig. S9) compared with that in HDM- treated mice. These results suggested that inhibition of EGFR activation increased the expression of claudin 1 and decreased MUC5AC, leading to reduced allergic airway inflammation.

\section{DISCUSSION}

As reported previously, $20-25 \%$ of airway epithelial cells for asthmatics are goblet cells ${ }^{25-27}$. As one of the key characteristic pathological changes of asthma, goblet-cell hyperplasia has been consistently observed in mild, moderate and severe stages of the disease. Also, mucous cell metaplasia is the foundation for mucus hypersecretion that can obstruct the airway lumen, which greatly exacerbates asthma ${ }^{3,15,28,29}$. Although epithelial 
a
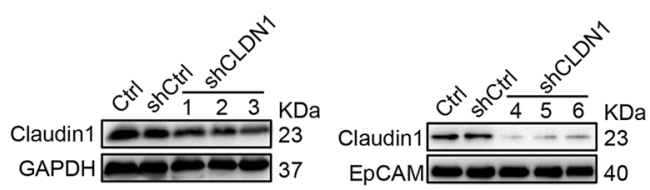
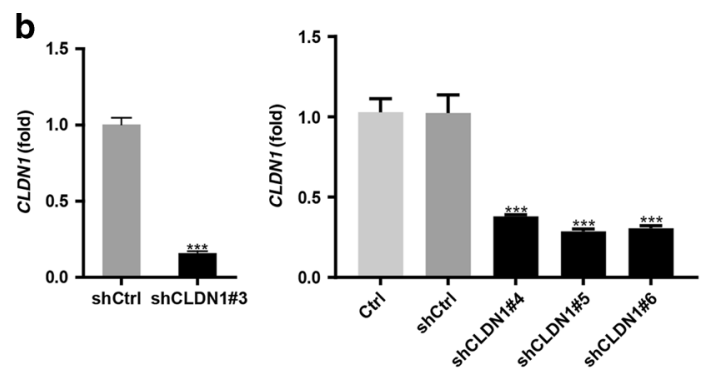
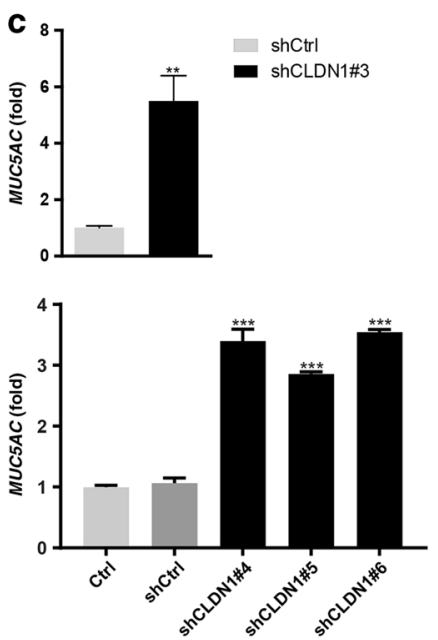

e
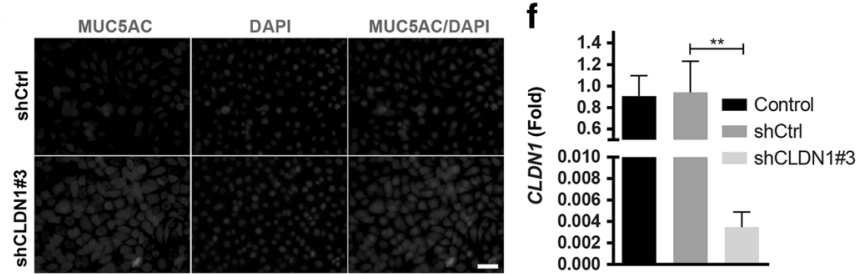

g

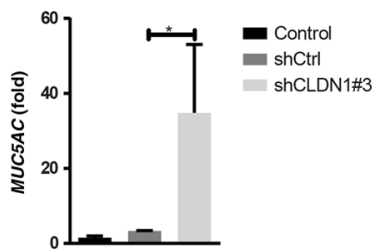

h
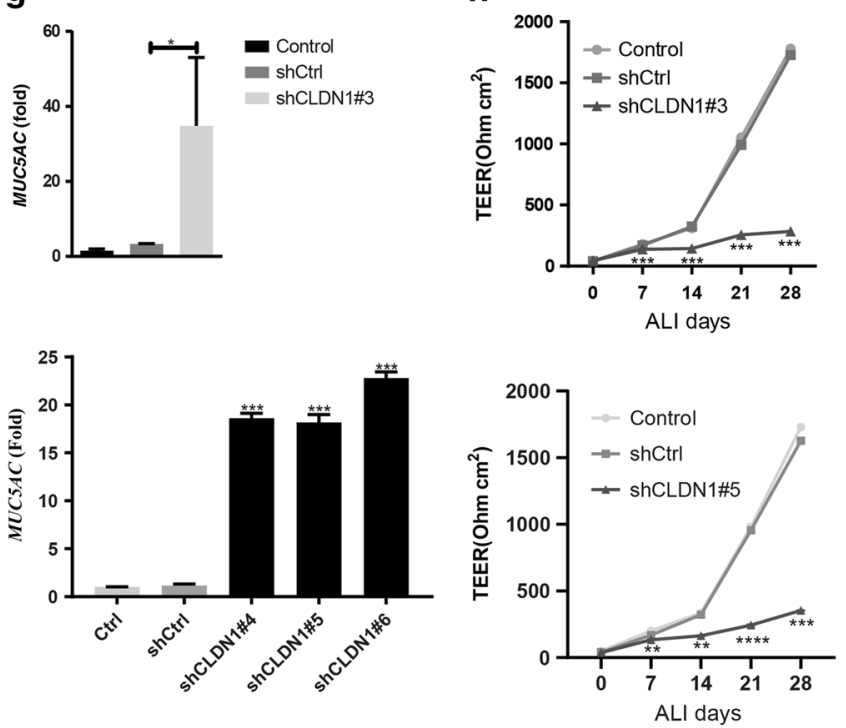

d
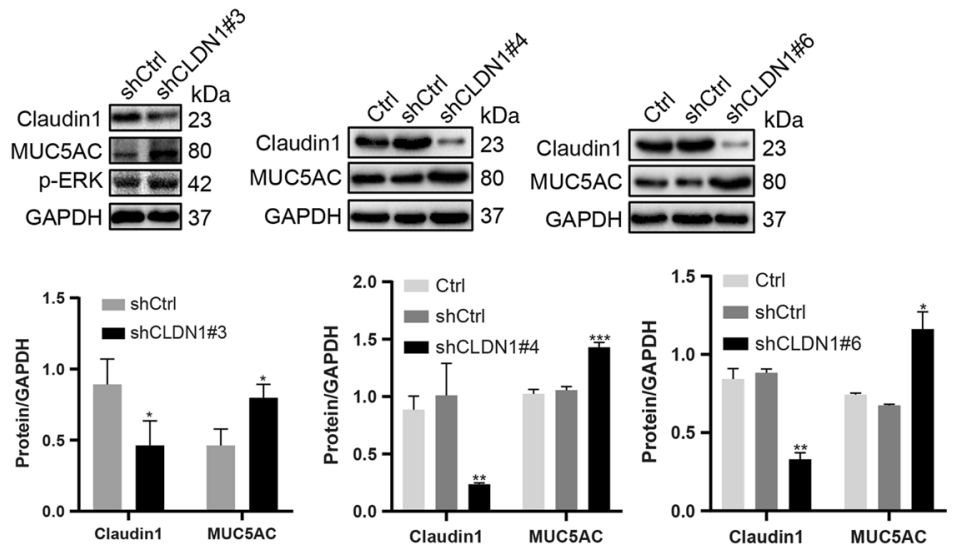
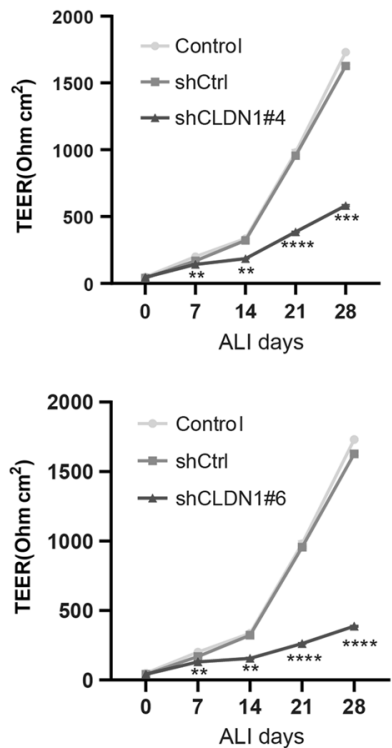

hyperplasia and metaplasia are well-known features of asthma, the basis for the altered epithelial phenotype has not been mechanistically determined.

The epithelial barrier function depends on cellular integrity, especially structures like $\mathrm{TJs}^{30}$. TJs establish cell-cell contact and cell polarity, and connect the differentiated ciliated and secretory cells, which maintain the integrity of the epithelial barrier ${ }^{31}$. By analyzing the GEO database, we found that claudin 1 was decreased both in the bronchial epithelium of patients with mild asthma and severe asthma. Moreover, the results of our subsequent experiments showed that downregulation of claudin1 significantly disturbed epithelial barrier function as 
Fig. 2 Claudin 1 knockdown promotes MUC5AC expression in 16HBE cells and ALI cultures. a The 16HBE cells were exposure to shRNA for claudin 1, after $48 \mathrm{~h}$, the expression of claudin 1 was detected by western blotting (representative blots from three experiments). $\mathbf{b}$ Quantitative PCR analyses of CLDN1 from 16HBE cells transfected with screened shCLDN1 $(n=6)$ or control shRNA $(n=6)$ for 24 h. c Quantitative PCR detection of MUC5AC mRNA levels in 16HBE cells transfected with shCLDN1 $(n=6)$ or shCtrl $(n=6)$. d Western blot analysis of claudin1, MUC5AC, and p-ERK from 16HBE cells transfected with shCLDN1 or shCtrl for $24 \mathrm{~h}$ (representative blots from three experiments). The relative intensities of claudin 1 and MUC5AC were evaluated by ChemiScope analysis software. e Immunofluorescence analysis of MUC5AC from $16 \mathrm{HBE}$ cells transfected with shCLDN1 or shCtrl for $24 \mathrm{~h}$ (Scale bar: $50 \mu \mathrm{m}$; original magnification: $\times 400$; representative images from three experiments). $\mathbf{f}, \mathbf{g}$ The mRNA expressions of CLDN1 and MUC5AC were detected with qRT-PCR after ALI culturing for 28 days ( $n=3$ per group). h Transepithelial electrical resistance measurements were carried out on days $0,7,14,21$, and 28 following cell differentiation at the air-liquid interface ( $n=3$ per group). Statistical comparisons were performed using two-way analysis of varianceA with Bonferroni's multiple comparison and unpaired two-tailed Student's $t$-test with Welch's correction (all data are represented as the mean \pm SD, ${ }^{*} P<0.05 ;{ }^{* * *} P<0.01$; $\left.{ }^{* * *} P<0.001 ;{ }^{* * * *} P<0.0001\right)$.

evidenced by a reduced TEER. Airway epithelial cells not only participate in the formation of the barrier function, but are also involved in mucus secretion, and claudin 1 plays a critical role in maintaining the epithelial barrier, raising the possibility that claudin1 may regulate mucus secretion. To confirm this hypothesis, we silenced claudin 1 in $16 \mathrm{HBE}$ cells, ALI cultures, and in mice lungs. The results showed that silencing of claudin 1 might alter the epithelial cell phenotype toward goblet cells by inducing MUC5AC expression. It has been reported that MUC5AC expression in asthma increases significantly and consistently ${ }^{3,32}$; moreover, MUC5AC is required for allergic airway hyperreactivity and may be a potential target for treating asthma and other lung diseases with this overproduced protein ${ }^{33}$. Notably, we found that claudin 1 downregulation increased the expression of p-ERK, and previous studies have shown that activation of the ERK signal pathway could induce MUC5AC ${ }^{34}$, so we speculated that claudin1 downregulation might induce MUC5AC by activating ERK signal pathway.

Damage to the barrier functions of the airway epithelium enhances mucosal permeability of asthmatic patients ${ }^{35}$. Because claudin 1 is pivotal for epithelial barrier function and MUC5AC induction, we further determined whether claudin1 downregulation was proportional to asthma exacerbation. First, our results suggested that claudin 1 expression negatively correlated with asthma severity. Second, the downregulation of claudin 1 exacerbated HDM-induced allergic airway inflammation. Finally, silencing of claudin 1 in vivo substantially increased the expression of MUC5AC in airway epithelial cells, which contributed to AHR augmentation in the HDM-induced mice asthma model. In summary, we suggest that excessive mucus production and allergic airway inflammation induced by claudin 1 downregulation might be associated with asthma exacerbation, and regulation of this process could be important in alleviating asthma.

Studies have shown that bronchial epithelial EGFR increased in asthma and more importantly, was correlated with disease severity $^{23,36}$. For the first time, our study found that EGFR ligands significantly decreased the expression of claudin1, among major junction proteins in bronchial epithelial cells and ALI cultures. We also found that IL-13, which has a disruptive effect on airway epithelial barrier function ${ }^{37}$, slightly reduced claudin 1 expression. However, it was noteworthy that IL-13 cooperated with HBEGF to further down-regulate the expression of claudin1. Moreover, AG1478 , a selective EGFR inhibitor, inhibited the decrease of claudin 1 expression induced by HBEGF and IL-13 in human bronchial epithelial cells. In the HDM-induced mice asthma model, erlotinib, as an EGFR antagonist, restored the expression of claudin1. These results might explain the mechanism of decreased claudin1 expression in asthma patients, and suggests that inhibition of EGFR activation may regulate the expression and function of claudin1.

Notably, epithelial EGFR signaling also contributes to goblet-cell hyperplasia and mucous hypersecretion in asthma ${ }^{19,24,38}$. In our study, EGFR ligands, which are elevated in samples from asthma patients $^{22}$, also induced the expression of MUC5AC in human airway epithelial cell lines and ALI cultures, and erlotinib reduced the MUC5AC levels in the HDM-induced asthma model. Moreover, EGFR activation contributed to the formation of "leaky" junctions and injured the epithelial barrier by reducing CLDN1 expression during ALI culturing. This also indirectly illustrated the correlation between the downregulation of claudin 1 expression and gobletcell metaplasia.

In our study, erlotinib alleviated airway allergic inflammation in the HDM-induced asthma mice model by reducing the number of EOS cells in blood and total cells in BALF, the levels of IL-4 and IL13 in BALF, lung inflammation infiltration and AHR. It has also been reported that gefitinib, an EGFR inhibitor, reduced the inflammatory cell counts and the levels of released cytokine concentrations (IL-4 and IL-13) in BALF, as well as eosinophil recruitment in the lungs and AHR in ovalbumin-induced mouse model of asthma ${ }^{39,40}$. These findings suggested that inhibition of the EGFR activation might alleviate airway allergic inflammation and AHR by restoring claudin1, and this might play a role in treating asthma.

In summary, claudin1 downregulation promoted MUC5AC expression, and exacerbated allergic airway inflammation and AHR. For the first time, we found that EGFR, activated by its ligands, downregulated claudin 1 expression in bronchial epithelial cells (Fig. 6). Restoring claudin 1 to maintain barrier integrity by inhibition of the EGFR cascade may be a novel and potential therapeutic strategy in the treatment of asthma.

\section{MATERIALS AND METHODS}

For a detailed description, please refer to supplementary sections.

\section{Reagents and chemicals}

The reagents used in this study were purchased as follows. Primers were ordered from Genscript (Nanjing, China). Erlotinib and AG1478 were purchased from Selleck (Shanghai, China). Lipopolysaccharides (LPS) and acetyl- $\beta$-methylcholine chloride were purchased from Sigma-Aldrich (Sigma-Aldrich China, Shanghai, China). TNF-a was obtained from R\&D Systems (Minneapolis, MN, USA). EGF, HBEGF, IL-1 $\beta$, and IL-13 were obtained from PeproTech (Rocky Hill, NJ, USA). HDM (Dermatophagoides pteronyssinus) was purchased from Greer Laboratories (Lenoir, NC, USA).

\section{Animal experiments}

Male BALB/c mice (Charles River, Beijing, China) were allocated randomly. The HDM-induced asthma model was established, and the effects of shCLDN1 and erlotinib were evaluated. For a complete description of the materials and methods used in the murine experiments, please refer to the supplementary sections.

Culture and differentiation of $16 \mathrm{HBE}$ cells Human bronchial epithelial cells (16HBE) were cultured in RPMI 1640 medium (Gibco, Gaithersburg, MD, USA) containing 10\% 
a
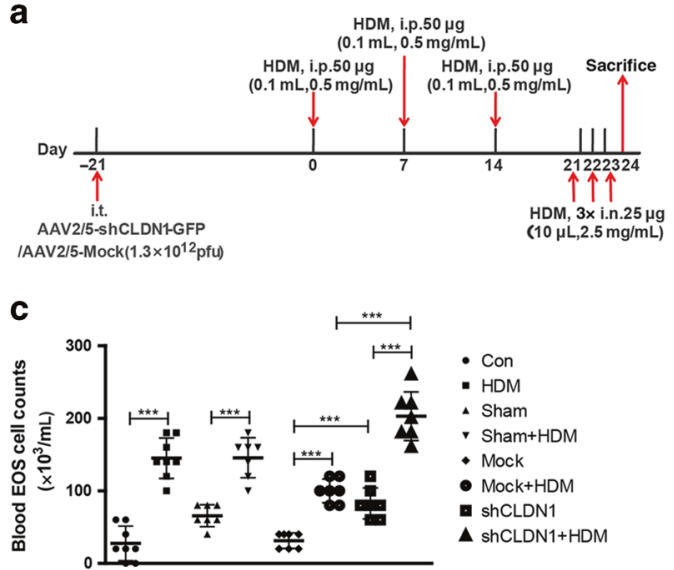

d

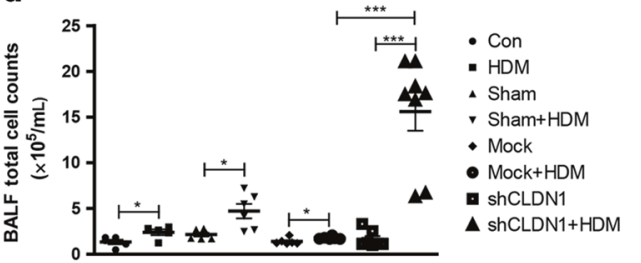

b
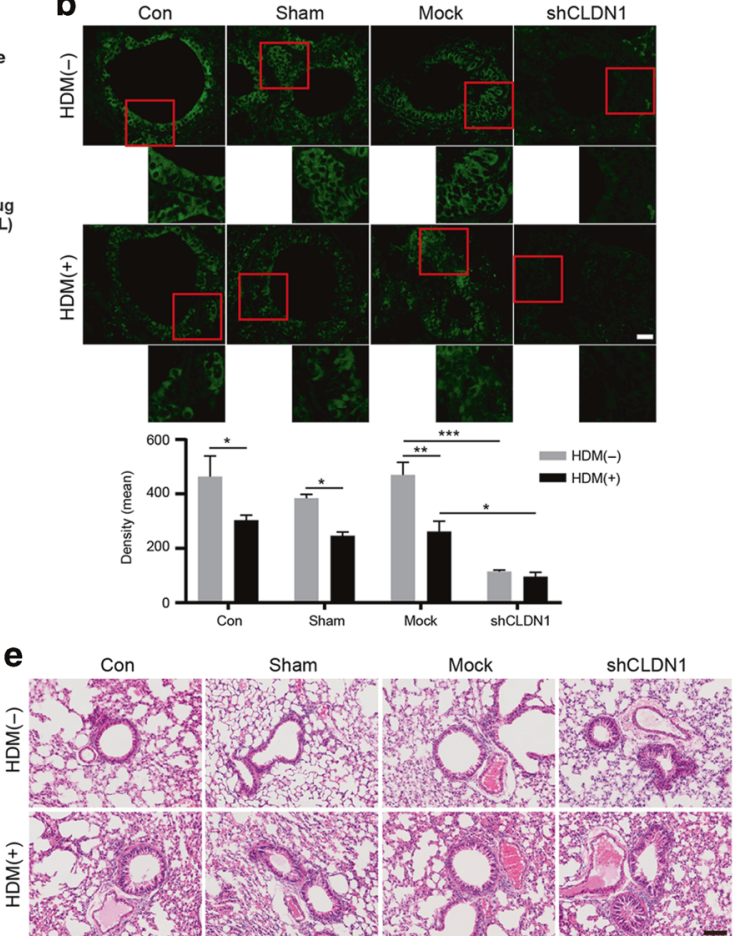

f

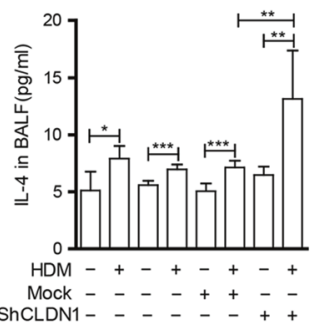

g

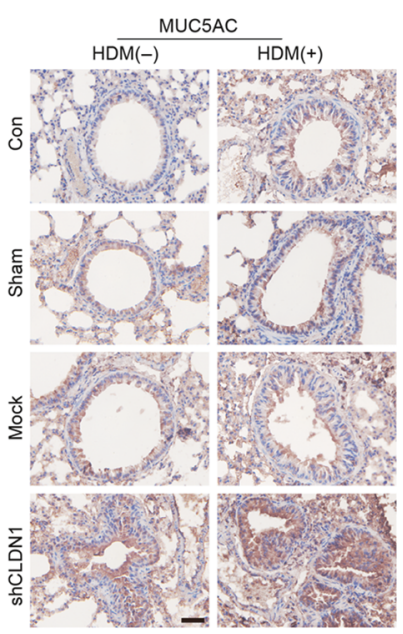

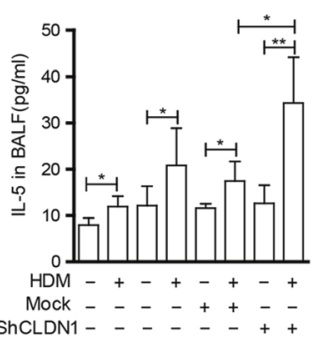

h
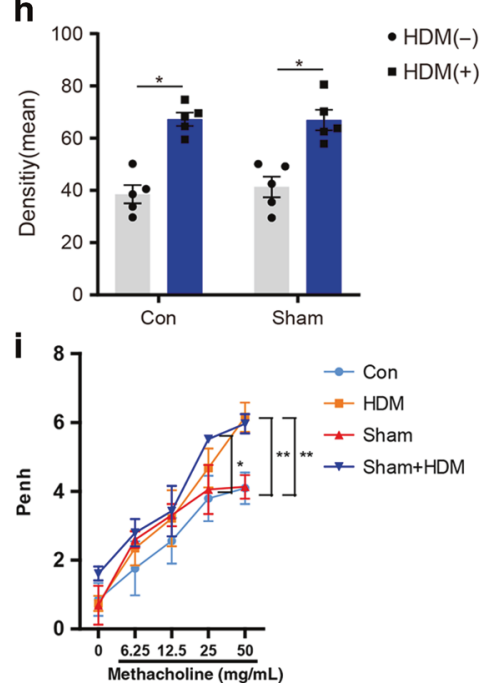
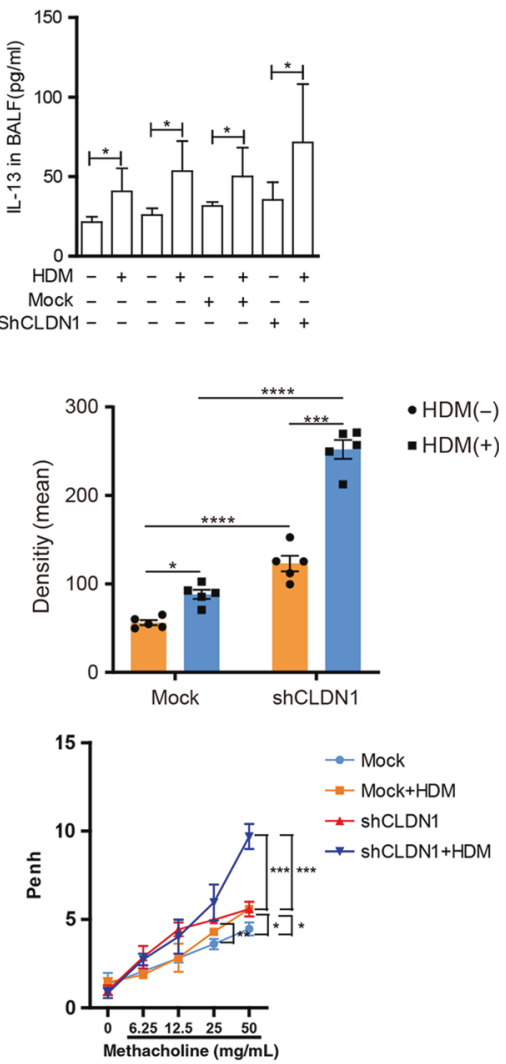

fetal bovine serum (Gibco, Gaithersburg, MD, USA) and differentiated for 28 days at an air-liquid interface (ALI) ${ }^{41}$. Differentiated cultures were treated basolaterally with human bronchial epithelial growth factor (HBEGF) at $10 \mathrm{ng} / \mathrm{mL}$.
Measurement of the transepithelial electrical resistance (TEER) TEER was measured with an epithelial volt ohm meter (EVOM) with a STX2 electrode (World Precision Instruments, Hitchin, Herts, UK). 
Fig. 3 Claudin1 downregulation exacerbates HDM-induced asthma. a Heterozygous adeno-associated virus (AAV) vector AAV2/5 comprising the sequence that silencing CLDN1 or negative control (AAV2/5-mock) were administered to mice through intratracheal injection and the HDM-induced asthma model was subsequently established. $\mathbf{b}$ The expression of claudin 1 was determined by immunofluorescence and the mean density of claudin 1 was analyzed by IPP software $(50 \mu \mathrm{m}$; original magnification: $\times 400 ; n=3)$. c Eosinophils in blood were counted with an Eosinophil Count kit $(n=7-8)$. d Cells in BALF were resuspended and counted with a Countstar automatic cell counter to obtain total cell counts $(n=5-8)$. e Hematoxylin and eosin -stained lung tissue sections $(100 \mu \mathrm{m} ;$ original magnification: $\times 200 ; n=3)$. $f$ The concentrations of IL-4, IL-5, and IL-13 in BALF were detected by ELISA $(n=6-8)$. g The expression of MUC5AC was determined by immunohistochemistry $(50 \mu \mathrm{m}$; original magnification: $\times 400 ; n=5)$. $\mathbf{h}$ The mean density of MUC5AC expression in Figure $\mathbf{g}$ was counted by IPP software. i Penh (enhanced pause) in MCh-challenged mice ( $n=4$ mice per condition). Statistical comparisons were performed using two-way analysis of variance with Bonferroni's multiple comparison and unpaired two-tailed Student's $t$-test with Welch's correction (****P<0.0001; ${ }^{* * *} P<0.001 ;{ }^{* * P}<0.01 ;{ }^{*} P<0.05 ;$ mean $\left.\pm \mathrm{SD}\right)$.

a

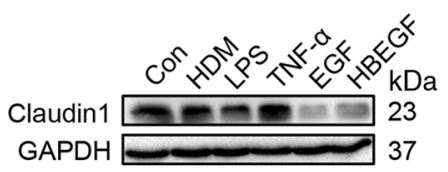

b

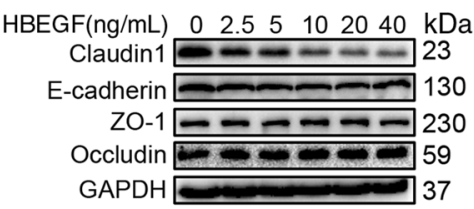

C

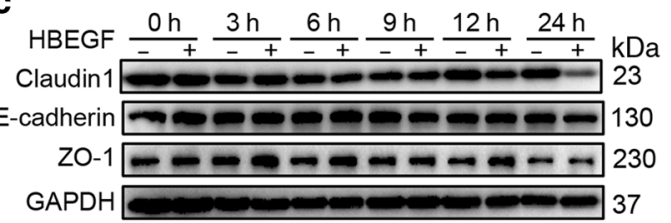

d

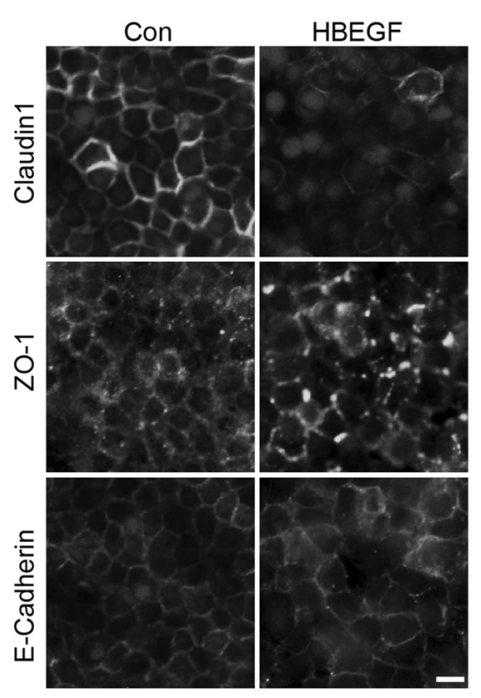

e

IL-13(ng/mL) $0103060100 \mathrm{kDa}$

Claudin1 - 23

GAPDH 37

g

HBEGF - - + +

$A G 1478(\mu \mathrm{M}) \quad 0 \quad 10 \quad 0 \quad 10 \mathrm{kDa}$

Claudin1 23

GAPDH

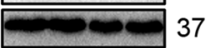

f

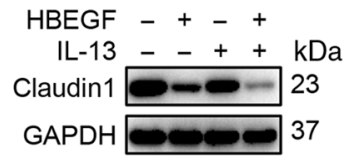

h

AG1478 _ + + + kDa

Claudin1 23

GAPDH 37

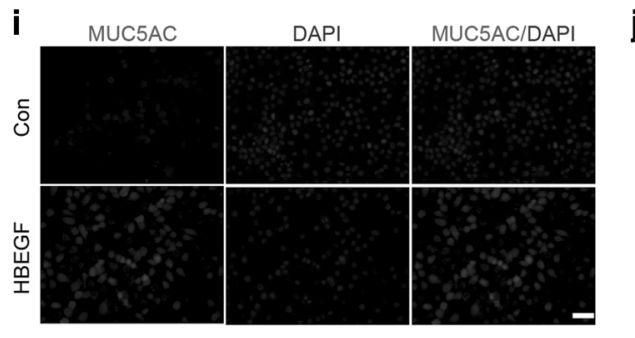

j HBEGF(ng/mL) $0 \quad 20 \mathrm{kDa}$ MUC5AC 80

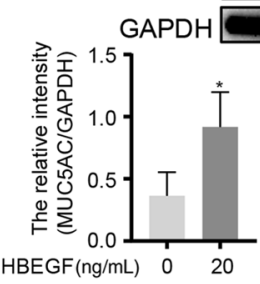

m

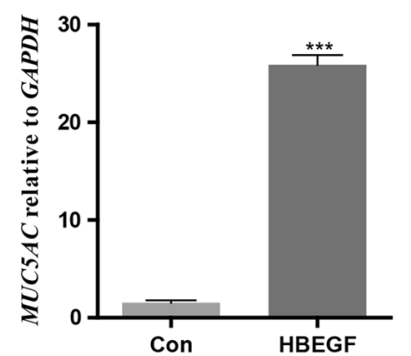

n

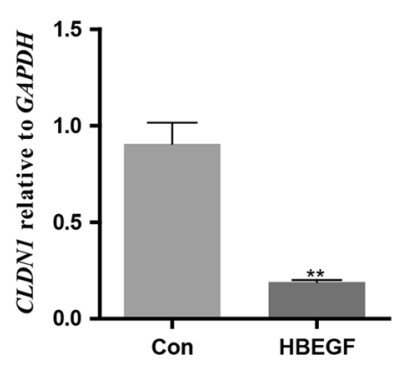

I

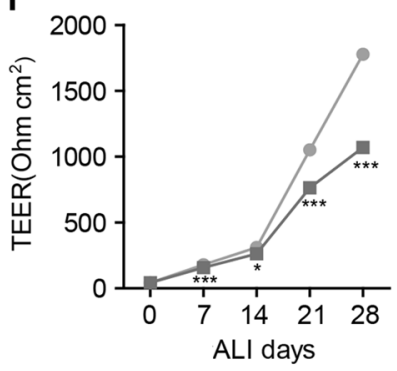

$\rightarrow$ Control

- HBEGF $(10 \mathrm{ng} / \mathrm{mL})$

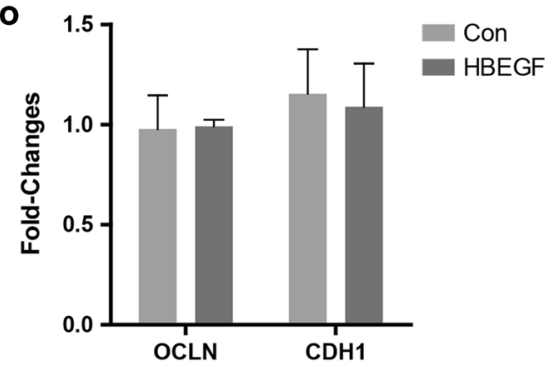

p

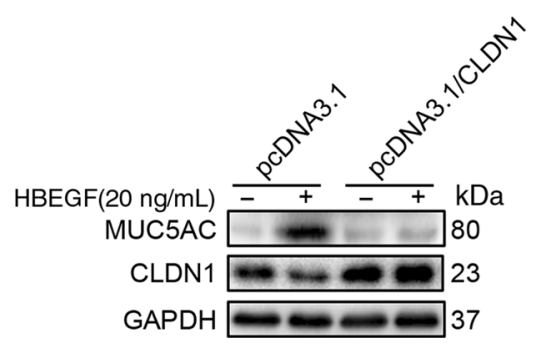


132

Fig. 4 EGFR activation promotes MUC5AC expression by reducing the expression of claudin1. a The 16HBE cells were stimulated respectively with HDM, LPS, TNF- $\alpha$, EGF, and HBEGF for $24 \mathrm{~h}$ (representative blots from three experiments). b $16 \mathrm{HBE}$ cells were stimulated with various concentrations of HBEGF for $24 \mathrm{~h}$, and the expressions of claudin1, E-cadherin, ZO-1, and occludin were detected by western blotting (representative blots from three experiments). c $16 \mathrm{HBE}$ cells were stimulated with HBEGF at indicated time points, and the expressions of claudin1, E-cadherin, and ZO-1 were detected by western blotting (representative blots from three experiments). d 16HBE cells were stimulated with HBEGF $(20 \mathrm{ng} / \mathrm{mL})$ for $24 \mathrm{~h}$, and the expressions of claudin 1 , ZO-1, and E-cadherin were detected by immunofluorescence (Scale bar: $50 \mu \mathrm{m}$; original magnification: $\times 400$; representative images from three experiments). e 16HBE cells were stimulated with various concentrations of IL-13 for $24 \mathrm{~h}$ (representative blots from three experiments). $\mathrm{f} 16 \mathrm{HBE}$ cells were stimulated respectively with HBEGF $(20 \mathrm{ng} / \mathrm{mL}), \mathrm{IL}-13(100 \mathrm{ng} / \mathrm{mL})$, and HBEGF $(20 \mathrm{ng} / \mathrm{mL})+\mathrm{IL}-13(100 \mathrm{ng} / \mathrm{mL})$ for $24 \mathrm{~h}$ (representative blots from three experiments). g, h $16 \mathrm{HBE}$ cells were incubated with AG1478 $(10 \mu \mathrm{M})$ or with their vehicle, $0.1 \%$ dimethyl sulfoxideas a control, for $1 \mathrm{~h}$. and were then treated with HBEGF or IL-13 for $24 \mathrm{~h}$ (representative blots from three experiments). i-k $16 \mathrm{HBE}$ cells were stimulated with $\mathrm{HBEGF}(20 \mathrm{ng} / \mathrm{mL})$ for $24 \mathrm{~h}$, and the expression of MUC5AC was detected, respectively, by immunofluorescence (Scale bar: $50 \mu \mathrm{m}$; original magnification: $\times 200$; representative images from three experiments), western blotting (representative blots from three experiments), and qPCR ( $n=6$ per group). The relative intensity of MUC5AC was assessed by ChemiScope analysis software. I Differentiated cultures were treated basolaterally with HBEGF $(10 \mathrm{ng} / \mathrm{mL})$, transepithelial electrical resistance measurements were carried out on days $0,7,14,21$, and 28 following cell differentiation at the air-liquid interface, $\mathbf{m}-\mathbf{o}$ The expressions of CLDN1, MUC5AC, CDH1, and OCLN in ALI cultures were determined by RT-PCR ( $n=3$ per condition). p 16HBE cells were transfected with pcDNA3.1-CLDN1 or empty vector. After $6 \mathrm{~h}$, the medium was replaced with RPMI 1640 containing $10 \%$ fetal bovine serum. After $24 \mathrm{~h}$ of continued culture, the cells were stimulated with or without HBEGF $(20 \mathrm{ng} / \mathrm{mL})$ for $24 \mathrm{~h}$, and MUC5AC expression was detected by western blotting. Statistical comparisons was performed using two-way analysis of variance with Bonferroni's multiple comparison and the unpaired two-tailed Student's $t$-test with Welch's correction $\left({ }^{* * *} P<0.001 ;{ }^{*} P<0.01 ;{ }^{*} P<0.05\right.$; mean \pm SD).

\section{Western blot analysis}

Cell or tissue lysates were subjected to SDS-PAGE and with the use of anti-claudin1(rabbit polyclonal IgG antibody; Abcam, Cambridge, UK), anti-MUC5AC (mouse monoclonal IgG1 antibody, clone 2-12M1; LifeSpan BioSciences, Seattle, WA, USA), anti-p-ERK(rabbit monoclonal IgG antibody, clone D13.14.4E) (Cell Signaling Technology, Boston, MA, USA); anti-Ecadherin (mouse monoclonal IgG2b antibody, clone \#180224; R\&D Systems, Minneapolis, MN, USA), anti-EpCAM, anti-ZO-1 and anti-occludin (rabbit polyclonal IgG antibody; Proteintech Group, Wuhan Sanying, Wuhan, China); and anti-glyceraldehye 3-phosphate dehydrogenase(GAPDH) (Bioss, Beijing, China) antibodies with horseradish peroxidase-electrochemiluminescence detection (Millipore, Burlington, MA, USA). The relative intensities of protein bands were analyzed by ChemiScope analysis software.

\section{Histology and immunohistochemistry}

Mice lung biopsy tissues were fixed with $10 \%$ formaldehyde and embedded in paraffin. Sections $(5 \mu \mathrm{m})$ were used for hematoxylin and eosin (H\&E) staining, diastase resistant (DPAS) staining (Servicebio, Wuhan, China), and immunohistochemical examinations. Sections were immunostained with antibodies against claudin1 (rabbit polyclonal IgG antibody, Abcam) and its isotype IgG (rabbit polyclonal IgG antibody; Wanleibio, Shenyang, China), MUC5AC (mouse monoclonal lgG1 antibody, clone SPM297; LifeSpan BioSciences), and pEGFR (rabbit monoclonal lgG1 antibody, clone D7A5; Cell Signaling Technology) followed standard protocols. The same microscopic magnification was used in all sample slides for comparisons, and in all cases, analysis was restricted to areas of well-orientated and structurally intact epithelia. The mean density of each protein was analyzed by Image-Pro Plus software (Media Cybernetics, Rockville, MD, USA).

\section{Immunofluorescence}

Proteins in $16 \mathrm{HBE}$ cells were detected by immunofluorescent staining with the use of antibodies against claudin1 (rabbit polyclonal IgG antibody; Abcam), E-cadherin (mouse monoclonal IgG2b antibody, clone \#180224; R\&D Systems), ZO-1(rabbit polyclonal IgG antibody; Proteintech Group) and MUC5AC (mouse monoclonal lgG1 antibody, clone SPM297; LifeSpan BioSciences) with a nuclear counterstaining. Staining was assessed with a live cell work station microscope (Carl Zeiss, Jena, Germany) with the use of identical settings between conditions. Claudin 1 expression in lung tissue sections was also detected and evaluated with a laser scanning confocal microscope (Carl Zeiss).
Quantitative real-time PCR

Total RNA was extracted from 16HBE cells using TRIzol reagent (Vazyme, Nanjing, China) before CDNA synthesis. Primers described in Supplementary Table 1 were used to quantify expression by RT-PCR (Applied Biosystems, Foster City, CA, USA) and the results were analyzed using the $\Delta \Delta C t$ method.

\section{ELISA}

Collected bronchoalveolar lavage fluid (BALF) and lung tissue homogenates were assessed for the expression of cytokines by using Ready-Set-Go! ELISA sets (eBioscience, Waltham, MA, USA) according to the manufacturer's instructions and with a Bio-Tek instrument (Bio-Tek Laboratories, Winooski, VT, USA).

shRNA construct and transfection shRNA construction was performed by Asia-vector Biotechnology (Shanghai, China) and Han-Bio (Shanghai, China). The four oligonucleotide sequences of the shRNA effectively targeting CLDN1 were respectively 5'-CGAAAATGGACATTGAGAT-3', 5'-GCAAAGU CUUUGACUCCUUTT-3', 5'- GCAGCACAUUGCAAGCAACTT-3', and $5^{\prime}$-GGUGCCCUACUUUGCUGUUTT- $3^{\prime}$. After $24 \mathrm{~h}$ in culture after seeding, shRNAs were transfected into $16 \mathrm{HBE}$ cells at a final concentration of $3 \mu \mathrm{g} / \mathrm{mL}$ using Lipofectamine 2000 (Life Technologies, Carlsbad, CA, USA) according to the manufacturer's protocol.

The overexpression of claudin 1 in 16HBE cells

The 16HBE cells were transfected with pcDNA3.1-CLDN1 (Hanbio, Shanghai, China) for the overexpression of claudin 1. The transfections were performed according to the manufacturer's instructions: $5 \mu \mathrm{g}$ of pcDNA3.1-CLDN1 or empty vector was used to transfect $70-80 \%$ confluent cells. Five microliters of Lipofectamine 2000 was used to transfect plasmid DNAs into $16 \mathrm{HBE}$ cells growing in serum-free opti-MEM media (Gibco, Gaithersburg, MD, USA). After $6 \mathrm{~h}$, the medium was replaced with RPMI 1640 containing $10 \%$ fetal bovine serum. After $24 \mathrm{~h}$ of continued culture, the cells were stimulated with or without HBEGF $(20 \mathrm{ng} / \mathrm{mL})$ for $24 \mathrm{~h}$, and MUC5AC expression was detected by western blotting.

\section{Gene expression dataset}

A gene expression dataset of primary bronchial epithelial cells from patients with severe asthma (accession no. GSE43696) was downloaded from the Gene Expression Omnibus (GEO) database (https://www.ncbi.nlm.nih.gov/geo/). Platforms of GEO datasets uesd GPL6480 for this dataset. 
a

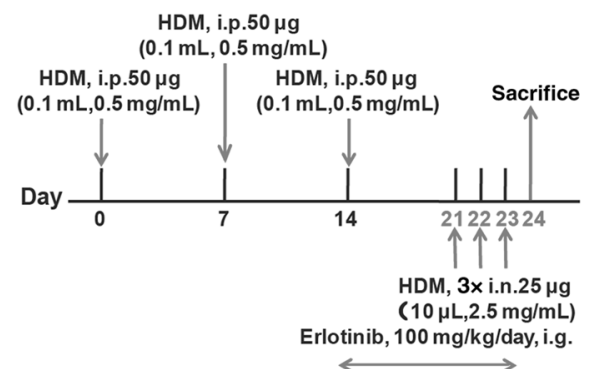

b

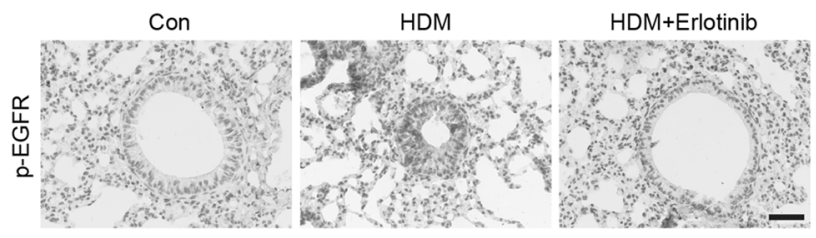

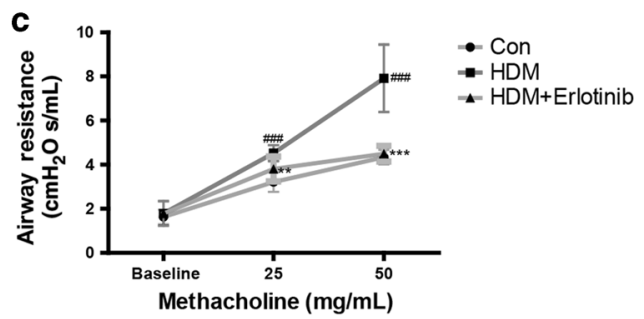
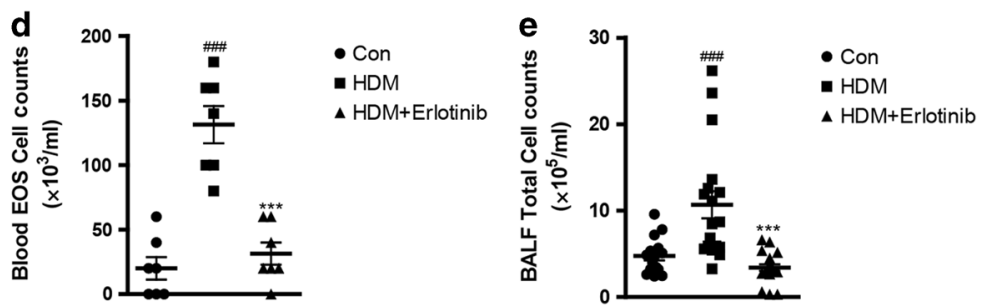

$\mathbf{f}$
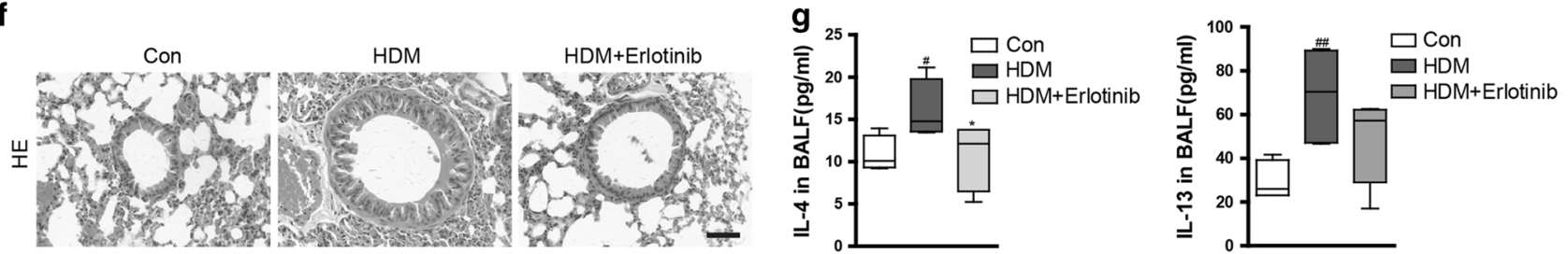

h
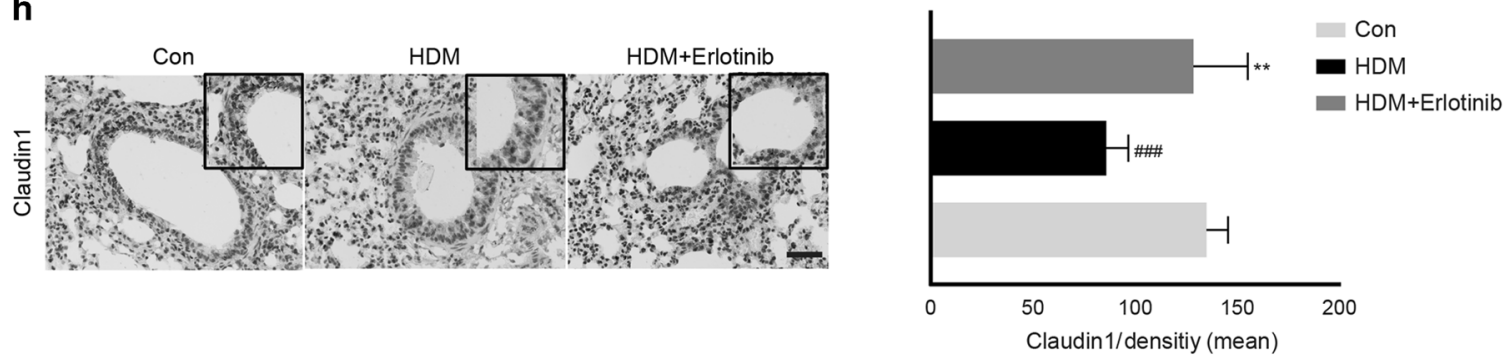

i
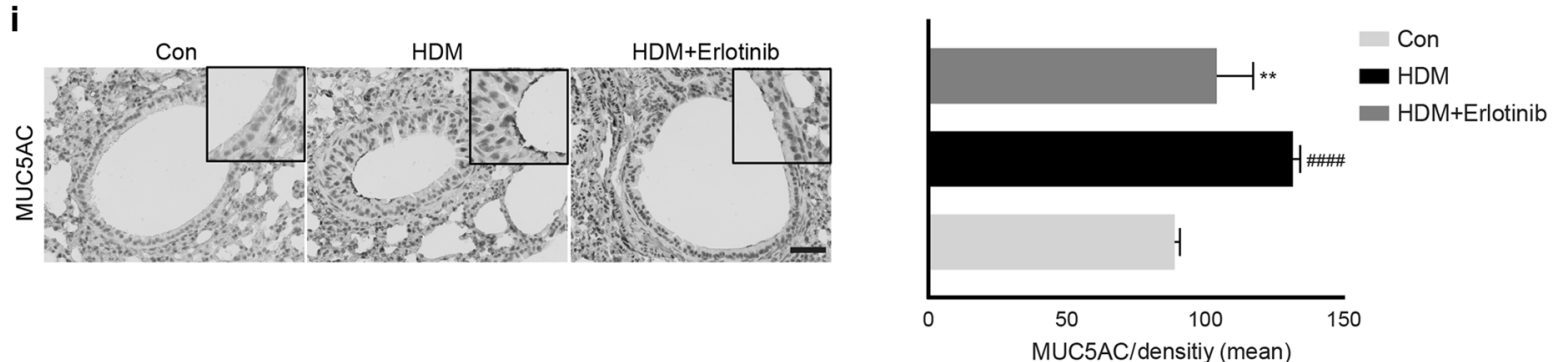

Fig. 5 Erlotinib alleviates mice allergic airway inflammation. a Flow charts of the HDM-induced asthma mouse model and erlotinib was administered during day 14-day 23. b The expression of p-EGFR was detected by immunohistochemistry (scale bar $50 \mu$ m; original magnification: $\times 400 ; n=3$ ). c Airway resistance was measured at baseline and in response to increasing doses of nebulized methacholine in anesthetized mice placed on a DSI Buxco PFT controller system. Data were derived from 3-5 animals per group. d, e The number of eosinophils in blood and total numbers of cells in BALF were counted. Data were derived from seven animals per group. $\mathbf{f}$ Hematoxylin and eosin -stained lung tissue sections ( $n=3$; scale bar: $50 \mu \mathrm{m}$; magnification: $\times 400)$. $\mathbf{g}$ The levels of IL-4 and IL-13 in BALF were detected by ELISA $(n=7) . \mathbf{h}$, $\mathbf{i}$ The expressions of claudin 1 and MUC5AC were detected by IHC and assessed by IPP software (scale bar: $50 \mu \mathrm{m} ;$ magnification: $\times 400 ; n=3$ ). Statistical comparisons were performed using one-way analysis of variance with Dunnett's test and two-way analysis of variance

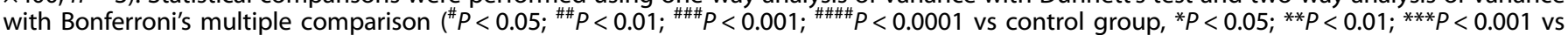
the HDM group).

Statistical analysis

Data are expressed as mean values \pm standard deviation (SD). Unpaired Student's $t$-test was used when comparing two groups. One-way analysis of variance with Dunnett's test or two-way analysis of variance with Bonferroni's multiple comparison was used to compare multiple groups. Statistical analysis was performed using Prism 5.00 or 8.00 software (GraphPad, San Diego, CA, USA). The differences were considered significant for $P<0.05$. 


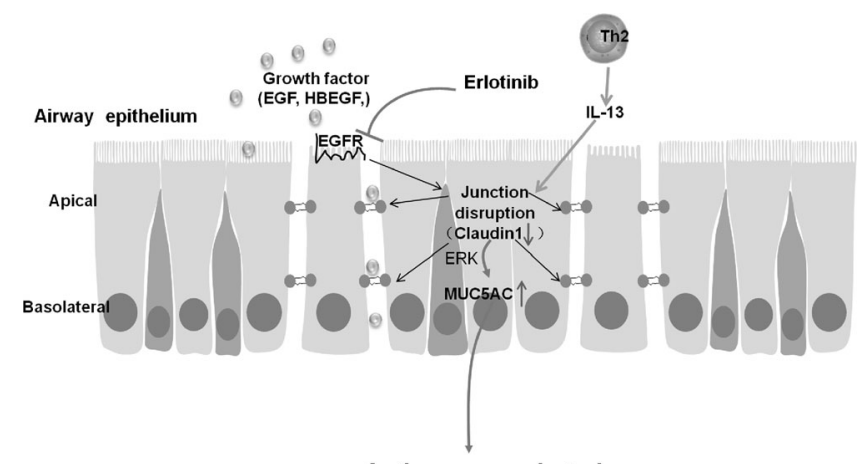

Asthma exacerbated

Fig. 6 Schematic model illustrating the molecular mechanism of claudin 1 downregulation exacerbates mice asthma. Our working hypothesis was that claudin 1 downregulation exacerbated HDMtriggered airway mucus secretionvia inducing the overexpression of MUC5AC. EGFR activation or transactivation in response to EGFR ligands (such as EGF or HBEGF) or IL-13 reduced expression of claudin1. Erlotinib, which inhibited EGFR signaling, reduced MUC5AC expression via restoring claudin 1 expression.

\section{ACKNOWLEDGEMENTS}

This work was supported by the National Natural Science Foundation of China (81473395, 81703733 and 81473390), the Priority Academic Program Development of Jiangsu Higher Education Institutions, the Natural Science Foundation of Jiangsu Province (BK20191414), Key Project of Jiangsu Administration of Traditional Chinese Medicine (ZD201902) and the Jiangsu Key Laboratory for Pharmacology and Safety Evaluation of Chinese Materia Medica (JKLPSE201603).

\section{AUTHOR CONTRIBUTIONS}

Z.R.J. and M.H. designed experiments. Z.R.J., K.F.B., P.W., X.R.Y., Y.H.Z., X.T.W., X.Y.W., W.Y.Y., and S.Q.W. performed the experiments. L.Y., L.Q.L., and P.W. assisted with the experiments of intratracheal injection. Z.R.J. wrote the manuscript. K.F.B., J.Z., and M.H. revised the manuscript. Y.Q.H. and M.H. evaluated the work progress.

\section{ADDITIONAL INFORMATION}

The online version of this article (https://doi.org/10.1038/s41385-020-0272-z) contains supplementary material, which is available to authorized users.

Competing interests: The authors declare no competing interests.

Publisher's note Springer Nature remains neutral with regard to jurisdictional claims in published maps and institutional affiliations.

\section{REFERENCES}

1. Erle, D. J. \& Sheppard, D. The cell biology of asthma. J. Cell Biol. 205, 621-631 (2014).

2. Evans, C. M. et al. Mucin is produced by clara cells in the proximal airways of antigen-challenged mice. Am. J. Respir. Cell Mol. Biol. 31, 382-394 (2004).

3. Ordonez, C. L. et al. Mild and moderate asthma is associated with airway goblet cell hyperplasia and abnormalities in mucin gene expression. Am. J. Respir. Crit. Care Med. 163, 517-523 (2001).

4. Fahy, J. V. Goblet cell and mucin gene abnormalities in asthma. Chest 122(6 Suppl), 320S-326S (2002).

5. Evans, C. M. et al. Mucus hypersecretion in asthma: causes and effects. Curr. Opin. Pulm. Med. 15, 4-11 (2009).

6. Gandhi, V. D. \& Vliagoftis, H. Airway epithelium interactions with aeroallergens: role of secreted cytokines and chemokines in innate immunity. Front. Immunol. 6, 147 (2015).

7. Georas, S. N. \& Rezaee, F. Epithelial barrier function: at the front line of asthma immunology and allergic airway inflammation. J. Allergy Clin. Immunol. 134, 509-520 (2014).

8. de Boer, W. I. et al. Altered expression of epithelial junctional proteins in atopic asthma: possible role in inflammation. Can. J. Physiol. Pharm. 86, 105-112 (2008).

9. Bucchieri, F. et al. Asthmatic bronchial epithelium is more susceptible to oxidantinduced apoptosis. Am. J. Respir. Cell Mol. Biol. 27, 179-185 (2002).
10. Holgate, S. T. Epithelium dysfunction in asthma. J. Allergy Clin. Immunol. 120, 1233-1244 (2007). quiz 1245-6.

11. Balda, M. S. \& Matter, K. Tight junctions and the regulation of gene expression. Biochim. Biophys. Acta 1788, 761-767 (2009).

12. Gunzel, D. \& Yu, A. S. Claudins and the modulation of tight junction permeability. Physiol. Rev. 93, 525-569 (2013).

13. Soini, Y. Claudins in lung diseases. Respir. Res. 12, 70 (2011).

14. Ichikawa-Tomikawa, N. et al. Possible involvement of tight junctions, extracellular matrix and nuclear receptors in epithelial differentiation. J. Biomed. Biotechnol. 2011, 253048 (2011).

15. Fahy, J. V. \& Dickey, B. F. Airway mucus function and dysfunction. N. Engl. J. Med. 363, 2233-2247 (2010).

16. Furuse, $M$. et al. A single gene product, claudin-1 or -2 , reconstitutes tight junction strands and recruits occludin in fibroblasts. J. Cell Biol. 143, 391-401 (1998).

17. Polosa, R. et al. Expression of c-erbB receptors and ligands in the bronchial epithelium of asthmatic subjects. J. Allergy Clin. Immunol. 109, 75-81 (2002).

18. Enomoto, Y. et al. Tissue remodeling induced by hypersecreted epidermal growth factor and amphiregulin in the airway after an acute asthma attack. J. Allergy Clin. Immunol. 124, 913-20 e1 (2009). -7.

19. Nadel, J. A. Role of epidermal growth factor receptor activation in regulating mucin synthesis. Respir. Res. 2, 85-89 (2001).

20. Zhu, L. et al. Rhinovirus-induced major airway mucin production involves a novel TLR3-EGFR-dependent pathway. Am. J. Respir. Cell Mol. Biol. 40, 610-619 (2009).

21. Kim, S. \& Nadel, J. A. Fibrinogen binding ICAM-1 promotes EGFR-dependent mucin production in human airway epithelial cells. Am. J. Physiol. Lung Cell Mol. Physiol. 297, L174-L183 (2009).

22. Amishima, M. et al. Expression of epidermal growth factor and epidermal growth factor receptor immunoreactivity in the asthmatic human airway. Am. J. Respir. Crit. Care Med. 157(6 Pt 1), 1907-1912 (1998).

23. Takeyama, K., Fahy, J. V. \& Nadel, J. A. Relationship of epidermal growth factor receptors to goblet cell production in human bronchi. Am. J. Respir. Crit. Care Med. 163, 511-516 (2001).

24. Le Cras, T. D. et al. Epithelial EGF receptor signaling mediates airway hyperreactivity and remodeling in a mouse model of chronic asthma. Am. J. Physiol. Lung Cell Mol. Physiol. 300, L414-L421 (2011).

25. Shimura, S. et al. Continuity of airway goblet cells and intraluminal mucus in the airways of patients with bronchial asthma. Eur. Respir. J. 9, 1395-1401 (1996).

26. Rogers, D. F. Airway goblet cells: responsive and adaptable front-line defenders. Eur. Respir. J. 7, 1690-1706 (1994).

27. Cohn, L. Mucus in chronic airway diseases: sorting out the sticky details. J. Clin. Invest 116, 306-308 (2006).

28. Innes, A. L. et al. Ex vivo sputum analysis reveals impairment of proteasedependent mucus degradation by plasma proteins in acute asthma. Am. J. Respir. Crit. Care Med. 180, 203-210 (2009).

29. Jenkins, H. A. et al. Histopathology of severe childhood asthma: a case series. Chest 124, 32-41 (2003).

30. Kojima, T. et al. Regulation of tight junctions in upper airway epithelium. Biomed. Res. Int. 2013, 947072 (2013).

31. Crystal, R. G. Airway basal cells. The "smoking gun" of chronic obstructive pulmonary disease. Am. J. Respir. Crit. Care Med 190, 1355-1362 (2014).

32. Kirkham, S. et al. Heterogeneity of airways mucus: variations in the amounts and glycoforms of the major oligomeric mucins MUC5AC and MUC5B. Biochem. J. 361 (Pt 3), 537-546 (2002).

33. Evans, C. M. et al. The polymeric mucin Muc5ac is required for allergic airway hyperreactivity. Nat. Commun. 6, 6281 (2015).

34. Liu, J. et al. Role of IL-13Ralpha2 in modulating IL-13-induced MUC5AC and ciliary changes in healthy and CRSwNP mucosa. Allergy 73, 1673-1685 (2018).

35. Gon, Y. \& Hashimoto, S. Role of airway epithelial barrier dysfunction in pathogenesis of asthma. Allergol. Int. 67, 12-17 (2018).

36. Liu, M. et al. Immune responses to self-antigens in asthma patients: clinical and immunopathological implications. Hum. Immunol. 73, 511-516 (2012).

37. Saatian, B. et al. Interleukin-4 and interleukin-13 cause barrier dysfunction in human airway epithelial cells. Tissue Barriers 1, e24333 (2013).

38. Nadel, J. A. \& Burgel, P. R. The role of epidermal growth factor in mucus production. Curr. Opin. Pharm. 1, 254-258 (2001).

39. Song, L. et al. The chronic and short-term effects of gefinitib on airway remodeling and inflammation in a mouse model of asthma. Cell Physiol. Biochem. 38, 194-206 (2016).

40. Hur, G. Y. et al. Potential use of an anticancer drug gefinitib, an EGFR inhibitor, on allergic airway inflammation. Exp. Mol. Med. 39, 367-375 (2007).

41. Hardyman, M. A. et al. TNF-alpha-mediated bronchial barrier disruption and regulation by src-family kinase activation. J. Allergy Clin. Immunol. 132, 665-675 e8 (2013). 